\title{
ИССЛЕДОВАНИЕ ЭФФЕКТИВНОСТИ КОМПЛЕКСНОГО НЕСТАЦИОНАРНОГО ВОЗДЕЙСТВИЯ НА ЗАЛЕЖЬ С ВЫСОКОВЯЗКОЙ НЕФТЬЮ
}

\author{
Поплыгин Владимир Валерьевич1, \\ poplygin@bk.ru
}

\section{Уирсигроч Мариан ${ }^{2}$,}

m.wiercigroch@abdn.ac.uk

1 Пермский национальный исследовательский политехнический университет, Россия, 616990, г. Пермь, Комсомольский пр., 29.

2 Центр Прикладной динамики, Соединенное Королевство Великобритании и Северной Ирландии, AB24 3UE, г. Абердин, улица Университета, 69.

\begin{abstract}
Актуальность. При стационарной закачке воды для поддержания пластового давления наблюдается опережающее обводнение добывающих скважин. Преждевременное обводнение связано с высокой послойной и зональной неоднородностью коллекторов, высокой вязкостью нефти, подошвенной водой, интенсивной закачкой воды. Использование циклического заводнения позволяет увеличить охват пласта воздействием и текущий коэффрициент извлечения нефти. Прирост в добыче нефтти от использования метода достигает 4 \%, снижается отбор воды из залежи.

Цель: исследовать эфффективность использования циклического заводнения на залежи с высоковязкой нефтью, оценить наиболее эффрективную длительность периодов работы и остановки нагнетательных и добывающих скважин для достижения наибольших приростов в добыче нестии.

Объект: участок турнейской карбонатной залежи с высоковязкой нефтью. Средняя обводненность продукции скважин на залежи составляет $77,1 \%$, при отборе от начальных извлекаемых запасов - 49 \%. Участок эксплуатируется одной нагнетательной скважиной, расположенной в центре, и девятью добывающими скважинами по периметру водонефтяного контакта. В рассматриваемых условиях необходимо внедрять методы увеличения охвата паста воздействием и снижения обводненности.

Методы: определение оптимальных технологий циклической закачки воды и комплексного нестационарного воздействия, гидродинамическое моделирование циклического воздействия на участке нефртяного месторождения.

Результаты. Рассмотрены различные варианты работы нагнетательной скважины при реализации циклического заводнения. Получено, что соотношение продолжительности работы и остановки скважины, при условии 100 \% компенсации, практически не влияет на результат моделирования. При циклической работе нагнетательной скважины увеличение добычи нефтти происходит на 3,6 \% по сравнению с базовым вариантом. Больший технологический эфрфект возможен при комплексном воздействии - периодической остановке как нагнетательных, так и добывающих скважин. В случае комплексного воздействия накопленная добыча несрти на участке залежи увеличивается на 10,6 \% по сравнению с базовым вариантом.
\end{abstract}

\section{Ключевые слова:}

Залежь нефти, обводненость, циклическое заводнение, нестационарное заводнение, нефтеотдача.

\section{Введение}

Для поддержания пластового давления в нефтяные залежи закачивают различные агенты, в основном воду. Закачка воды в скважины позволяет достигать высоких значений коэффициентов нефтеотдачи пластов [1-5], препятствовать деформациям горных пород [6]. Вместе с тем при стационарной закачке наблюдается опережающее обводнение добывающих скважин из-за прорыва воды по более проницаемым каналам [7-10]. Для повышения эффективности эксплуатации залежей, изменения направления фильтрационных потоков, вовлечение в разработку ранее не дренируемых запасов используют различные технологии, в том числе закачку агентов повышенной вязкости, строительство новых скважин или боковых стволов и др. [11]. Одним из эффективных методов увеличения охвата пласта воздействием, снижения темпов опережающего обводнения добывающих скважин является циклическая закачка воды [12]. Промысловый опыт внедрения циклической закачки или нестационарного заводнения показывает существенный прирост уровней добычи нефти и коэффициентов нефтеизвлече- ния при минимальных затратах на внедрение технологии [13-17].

Выявлено, что эффективность нестационарного воздействия увеличивается с ростом неоднородности коллекторов, вязкости пластовой нефти, проницаемости отдельных прослоев и др. [18-22].

В Пермском крае на части объектов разработки наблюдается опережение обводнения скважин над выработкой извлекаемых запасов нефти (НИЗ). Наиболее существенное отличие между обводнением и выработкой НИЗ имеется на залежах с высоковязкой нефтью. Значительная часть залежей с высоковязкой нефтью в Пермском крае приурочена к Ножовской группе месторождений. Средние значения обводненности и отбора от НИЗ по турнейским объектам Ножовской группы месторождений составляют 57 и $37 \%$, соответственно.

\section{Объект исследований}

Рассмотрим одно из поднятий турнейской залежи месторождения Ножовской группы. Месторождение разрабатывается с 1994 г. Основные геолого-физические параметры и показатели разработки залежи приведены в табл. 1. 
Таблица 1. Основные геолого-физические параметры и показатели разработки залежи

Table 1. The main geological and physical parameters and deposits development indicators

\begin{tabular}{|c|c|c|}
\hline Параметр/Parameter & $\begin{array}{l}\text { Ед. измер. } \\
\text { Units }\end{array}$ & $\begin{array}{c}\text { Значение } \\
\text { Value }\end{array}$ \\
\hline $\begin{array}{l}\text { Проницаемость по керну } \\
\text { Core permeability } \\
\end{array}$ & $\begin{array}{l}\text { мкм }^{2} \\
\text { micron }^{2}\end{array}$ & 0,035 \\
\hline \begin{tabular}{|l|} 
Коэффициент песчанистости \\
Sandiness coefficient \\
\end{tabular} & $\begin{array}{c}\text { доли ед. } \\
\text { unit fraction }\end{array}$ & 0,574 \\
\hline \begin{tabular}{|l} 
Коэффициент расчлененности \\
Number of permeable intervals \\
\end{tabular} & $\begin{array}{c}\text { доли ед. } \\
\text { unit fraction }\end{array}$ & 4,33 \\
\hline $\begin{array}{l}\text { Вязкость нефти в пластовых условиях } \\
\text { Reservoir oil viscosity }\end{array}$ & $\begin{array}{l}\mathrm{m} \Pi a^{*} \mathrm{c} \\
\mathrm{mPa} \mathrm{s}^{\mathrm{s}}\end{array}$ & 42 \\
\hline $\begin{array}{l}\text { Вязкость воды в пластовых условиях } \\
\text { Reservoir water viscosity }\end{array}$ & $\begin{array}{l}\mathrm{M} \mathrm{a}^{*} \mathrm{c} \\
\mathrm{mPa} * \mathrm{~s}\end{array}$ & 1,44 \\
\hline \begin{tabular}{|l|} 
Отбор от НИЗ \\
Oil recovery \\
\end{tabular} & $\%$ & 49 \\
\hline $\begin{array}{l}\text { Обводненность } \\
\text { Water cut }\end{array}$ & $\%$ & 77,1 \\
\hline
\end{tabular}

Из табл. 1 следует, что обводненность существенно больше отбора от начальных извлекаемых запасов нефти. Данная ситуация обусловлена совокупностью факторов: высокой вязкостью пластовой нефти, расчлененностью залежи, существенным отличием проницаемостей пропластков. Дебиты скважин по нефти на участке изменяются в пределах 2,2...4,6 т/сут, по жидкости $4,5 \ldots 16,5 \mathrm{~m}^{3} /$ сут. Забойные давления на добывающих скважинах изменяются в пределах 6,1...14,9 МПа. Приёмистость нагнетательной скважины $30 \mathrm{~m}^{3} /$ сут.

Размещение скважин на рассматриваемом поднятии представляет собой элемент разработки с одной нагнетательной скважиной в центре и девятью скважинами по периметру водонефтяного контакта (рис. 1). В настоящее время действующими являются 4 добывающих скважины, остальные переведены в контрольный фонд, законсервированы, 1 скважина ликвидирована.

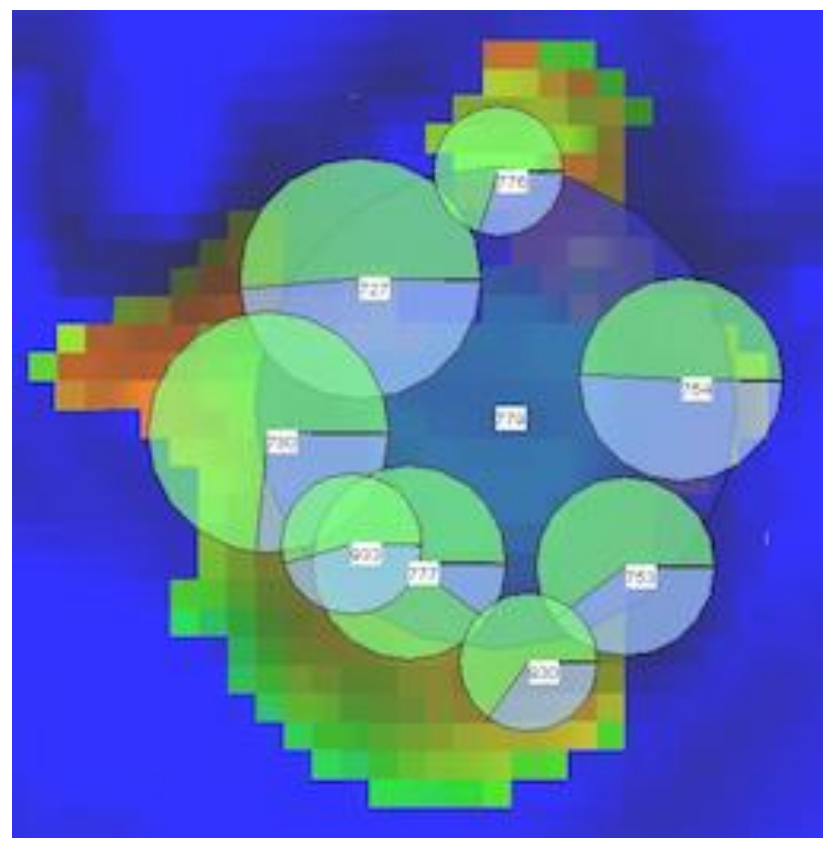

Puc. 1. Карта накопленных отборов и закачки на поднятии Fig. 1. Map of cumulative oil production and water injection

\section{Проектирование нестационарного воздействия}

При анализе движения фильтрационных потоков на поднятии отмечено, что значительная доля закачиваемой воды движется по более проницаемым каналам фильтрации от нагнетательной скважины к добывающей (рис. 2).

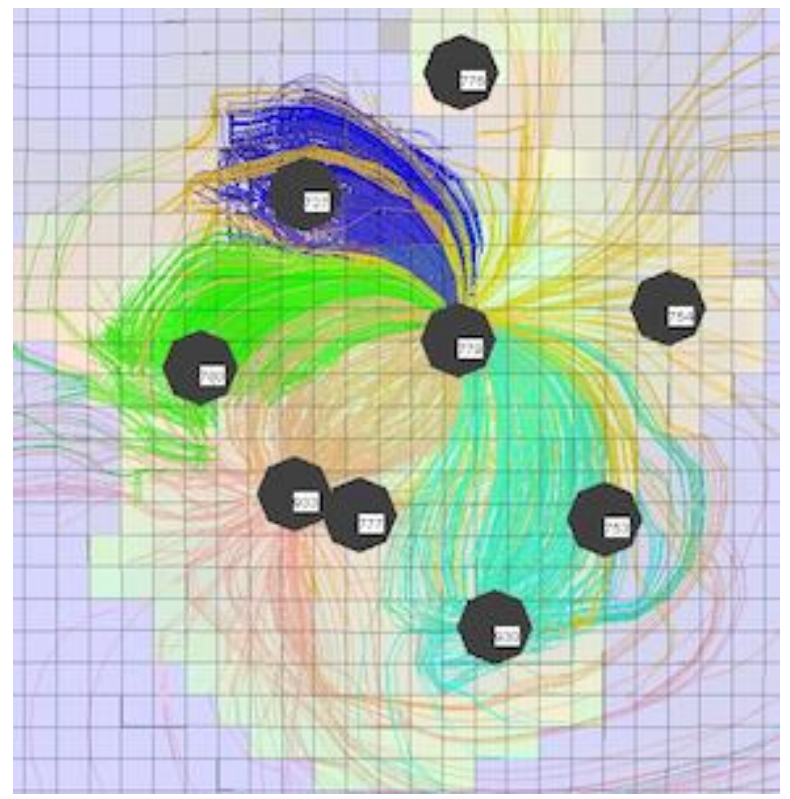

Рис. 2. Направления фильтрационных потоков на участке залежи

Fig. 2. Direction of filtration flows in the area of deposits

Для увеличения охвата пласта воздействием, вытеснения нефти из блоков коллектора и повышения эффективности разработки рассматриваемого участка залежи предлагается использовать технологию нестационарного заводнения. В специальной литературе отмечено, что необходимо учитывать фильтрационные параметры залежи для оценки оптимального времени остановки нагнетательных скважин [23] и чем больше проницаемость коллектора, тем больше должна быть величина полупериодов нагнетания [24].

В [25] приведена формула для определения времени полуциклов работы нагнетательной скважины:

$$
T=\frac{l^{2} \cdot m \cdot C \cdot \mu}{2 k},
$$

где $T$ - длительность полуцикла нестационарного воздействия; $l$ - среднее расстояние между нагнетательными и добывающими скважинами; $\mathrm{m}$ - пористость; $C$ - коэффициент сжимаемости породы и жидкости; $\mu$ - динамическая вязкость пластовой нефти; $k$ - проницаемость.

Для рассматриваемого поднятия длительность полуцикла, определенная по представленной формуле, приближается к 180 сут.

В $[26,27]$ показано, что наибольший эффект при реализации циклического заводнения достигается при соотношении времени работы и остановки нагнетательных скважин в интервале $1 / 2 \ldots 2 / 3$. Выполнено гидродинамическое моделирование нестационарного воздействия для рассматриваемого участка с текущего момента времени до 01.01.2030 в программном комплексе Tempest More (табл. 2). 
Таблица 2. Результаты моделирования ииклической закачки на участке залежи

Table 2. Results of simulation of cyclic injection in the area of deposits

\begin{tabular}{|c|c|c|c|c|}
\hline \multirow{3}{*}{$\begin{array}{l}\text { № вари- } \\
\text { aнта } \\
\text { Number }\end{array}$} & \multirow{3}{*}{$\begin{array}{c}\text { Продолжитель- } \\
\text { ность работы } \\
\text { нагнетательной } \\
\text { скважины, сут } \\
\text { Injection well } \\
\text { operation time, } \\
\text { days }\end{array}$} & \multirow{3}{*}{$\begin{array}{c}\text { Продолжитель- } \\
\text { ность остановки } \\
\text { нагнетательной } \\
\text { скважины, сут } \\
\text { Time to stop the } \\
\text { injection well, } \\
\text { days }\end{array}$} & \multicolumn{2}{|c|}{$\begin{array}{c}\text { Результаты на } \\
\text { Results for } \\
01.01 .2030\end{array}$} \\
\hline & & & $\begin{array}{l}\circ 0 \\
00 \\
\hat{\theta}\end{array}$ & 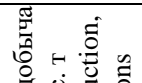 \\
\hline & & & 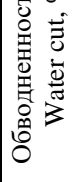 & 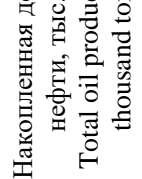 \\
\hline $\begin{array}{l}\text { Базовый } \\
\text { Base }\end{array}$ & - & - & 78,6 & 233,98 \\
\hline 1 & 30 & 30 & \multirow{3}{*}{70,6} & 242,51 \\
\hline 2 & 60 & 60 & & 242,50 \\
\hline 3 & 180 & 180 & & 242,49 \\
\hline 4 & 30 & 60 & 70,8 & 242,42 \\
\hline 5 & 60 & 180 & \begin{tabular}{|l|}
70,7 \\
\end{tabular} & 242,42 \\
\hline
\end{tabular}

Результаты моделирования (табл. 2) предполагают эффективность использования нестационарного воздействия в виде циклического заводнения. К 2030 г. ожидаемый прирост накопленной добычи нефти достигнет 3,6 \% при снижении обводненности на $8 \%$. Однако существенной разницы между результатами моделирования на различных режимах работы нагнетательной скважины не выявлено. Очевидно, что в текущих условиях наиболее технологически эффективным окажется вид нестационарного воздействия более удобный для реализации производственным подразделением.

В $[28,29]$ отмечена эффективность комплексного воздействия на залежь нефти с помощью циклической работы как нагнетательных, так и добывающих скважин. При таком воздействии происходит более интенсивное перераспределение фильтрационных потоков и давлений в пласте и, как следствие, увеличиваются притоки к добывающим скважинам.

Результаты моделирования комплексного нестационарного воздействия представлены в табл. 3.

Согласно табл. 3, комплексное нестационарное воздействие приводит к существенно большему приросту в добыче нефти на участке залежи. Максимальный прирост в добыче нефти в первом варианте составляет 10,6 \% по сравнению с базовым вариантом.

\section{СПИСОК ЛИТЕРАТУРЫ}

1. Ning J. Effects of different types of reservoir allocation and injection factors and model analysis// AIP Conference Proceedings. - 9 November 2018. - V. 2036. - Paper 030043.

2. Study on the influence of injection/production type and well pattern and spacing on producing degree of thin and poor layers / S. Liang, Y. Liu, N. Zhang, B. Zeng // Journal of Petroleum Exploration and Production Technology. - 1 September 2018. - V. 8. - Iss. 3. - P. 861-870.

3. Optimization of waterflood designs and operations using streamline simulation: Application to an oil field in the North German basin / F. Uzoechina, K.N. Awemo, A.E. Pieve La Rosa, C. Spyrou, S.K. Khataniar // Oil Gas European Magazine. - September 2018. V. 44. - Iss. 3. - P. 138-145.

4. A new method for development evaluation of waterflooding reservoirs / J. Feng, R. Luo, W. Tang, H. Zhang, T. Liu, D. Wang // Springer
Таблица 3. Результаты моделирования нестационарного гидродинамического воздействия на участке залежи

Table 3. Results of simulation of non-stationary hydrodynamic effects on the site of deposits

\begin{tabular}{|c|c|c|c|c|c|c|}
\hline \multirow[b]{2}{*}{ 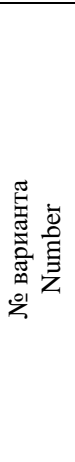 } & \multirow{2}{*}{ 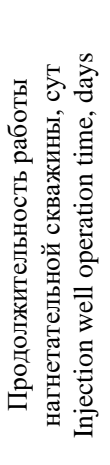 } & \multirow{2}{*}{ 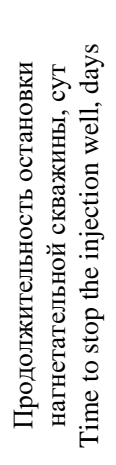 } & \multirow{2}{*}{ 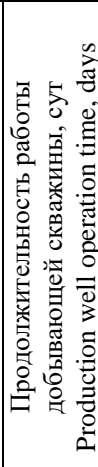 } & \multirow{2}{*}{ 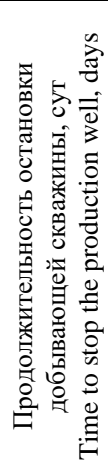 } & \multicolumn{2}{|c|}{$\begin{array}{c}\text { Результаты на } \\
\text { Results for } \\
01.01 .2030 \\
\end{array}$} \\
\hline & & & & & 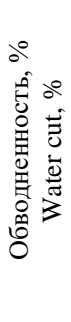 & 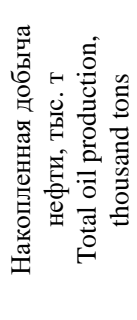 \\
\hline 1 & 30 & 60 & 30 & 30 & 74,3 & 258,79 \\
\hline 2 & 30 & 30 & 30 & 30 & 74,5 & 258,74 \\
\hline 3 & 60 & 60 & 60 & 60 & 73,3 & 256,3 \\
\hline
\end{tabular}

\section{Выводы}

1. Циклическое заводнение - эффективный способ увеличения коэффициента извлечения нефти на карбонатных залежах с высоковязкой нефтью. Согласно результатам исследования, при реализации технологии текущий коэффициент извлечения нефти увеличивается практически на $4 \%$ через десять лет за счет внедрения воды в ранее незатронутые дренированием участки залежи.

2. Наибольшую эффективность показал вид циклической закачки с временем работы и остановки нагнетательных скважин 30 сут. Разница в технологической эффективности между вариантами с различной длительностью полуциклов работы нагнетательной скважины несущественна.

3. Комплексное нестационарное воздействие (с остановками и добывающих, и нагнетательных скважин) позволяет увеличить текущий коэффициент извлечения нефти на 10,6 \% по сравнению со стационарным воздействием.

Исследование выполнено при финансовой поддержке Правительства Пермского края в рамках научного проекта No C-26/786 om 21.12 .20172

Series in Geomechanics and Geoengineering. - 2019. - Iss. 216039. P. 1414-1422.

5. Experimental investigation on water flooding and continued EOR techniques in buried-hill metamorphic fractured reservoirs / J. Wang, H. Liu, J. Zhang, Q. Meng, H. Liu, L. Ge, Z. Zhu, C. Liu // Journal of Petroleum Science and Engineering. - December 2018. - V. 171. P. 529-541.

6. Bataee M., Irawan S. Porosity and permeability alteration around wellbore during injection process // International Journal of Geomechanics. - February 2018. - V. 18. - Iss. 2. - Paper 04017145.

7. An approach for determining the water injection pressure of lowpermeability reservoirs / W. Lyu, L. Zeng, M. Chen, D. Qiao, J. Fan, D. Xia // Energy Exploration and Exploitation. - 1 September 2018. V. 36. - Iss. 5. - P. 1210-1228.

8. A method to calculate reasonable water injection rate for $\mathrm{M}$ oilfield / K. Yu, K. Li, Q. Li, K. Li, F. Yang // Journal of Petroleum 
Exploration and Production Technology. - 1 December 2017. - V. 7. Iss. 4. - P. 1003-1010.

9. Ogbeiwi P., Aladeitan Y., Udebhulu D. An approach to waterflood optimization: case study of the reservoir X // Journal of Petroleum Exploration and Production Technology. - 1 March 2018. - V. 8. Iss. 1. - P. 271-289.

10. Optimizing recovery for waterflooding under dynamic induced fracturing conditions / P.J. Van Den Hoek, R. Al-Masfry, D. Zwarts, J.D. Jansen, B. Hustedt, L. Van Schijndel // SPE Reservoir Evaluation and Engineering. - October 2009. - V. 12. - Iss. 5. - P. 671-682.

11. Optimization of horizontal injection and production pattern / Z.-F. Ling, Y.-L. Hu, B.-Z. Li, L.-J. Wang // Petroleum Exploration and Development. - February 2007. - V. 34. - Iss. 1. - P. 65-72.

12. Yang L. Water Injection Study in a Block Cycle // IOP Conference Series: Earth and Environmental Science. - 17 July 2018. - V. 170. Iss. 2. - Paper 022038.

13. Савенок О.В., Поварова Л.В., Гаскаров Н.Р. Эффективность химических методов стимуляции пласта и нестационарного циклического заводнения на Вынгапуровском месторождении // Булатовские чтения. - 2018. - Т. 2. - № 2. - С. 146-151.

14. Тараканова О.Э., Галиуллин М.М., Дубовецкая Н.В. Нестационарное заводнение как способ повышения эффективности реализованной системы поддержания пластового давления // Нефтяное хозяйство. - 2013. - № 11. - С. 49-53.

15. Чикиров Р.Р., Мамчистова Е.И., Чикиров Р.Р. Нестационарное заводнение как один из методов увеличения нефтеотдачи пластов с повышенной вязкостью нефти // Успехи современного естествознания. - 2018. - № 8. - С. 191-196.

16. Гуляев В.Н., Киприн И.И., Захарова Н.П. Применение технологии нестационарного воздействия для увеличения КИН участков с трудноизвлекаемыми запасами // Геология, геофизика и разработка нефтяных и газовых месторождений. - 2015. - № 10. - С. 39-44.

17. Циклическое заводнение бобриковского горизонта Сабанчинского нефтяного месторождения / А.Ф. Яртиев, А.Г. Хабибрахманов, В.Б. Подавалов, А.И. Бакиров // Нефтяное хозяйство. - 2017. № 3. - C. $85-87$.

18. Применение нестационарного заводнения на залежах высоковязкой нефти с коллектором двойной проницаемости. Теория / И.В. Владимиров, Э.М. Велиев, Э.М. Альмухаметова, Д.Т. Абилхаиров // Проблемы сбора, подготовки и транспорта нефти и нефтепродуктов. - 2014. - № 4 (98). - С. 16-25.

19. Велиев Э.М. Исследование зависимости эффективности технологии нестационарного заводнения от продолжительности полупериода работы/простоя нагнетательных скважин в цикле при разработке высокопродуктивных неоднородных коллекторов маловязкой нефти // Проблемы сбора, подготовки и транспорта нефти и нефтепродуктов. - 2015. - № 2 (100). - С. 46-56.
20. Чумаков Г.Н. Вероятностная оценка эффективности применения метода циклической закачки жидкости в пласт // Вестник Пермского национального исследовательского политехнического университета. Геология. Нефтегазовое и горное дело. - 2014. T. 13. - № 13. - С. 49-58.

21. Пятибратов П.В., Аубакиров А.Р. Оценка влияния анизотропии пласта по проницаемости на эффективность циклического заводнения // Экспозиция. Нефть. Газ. - 2016. - № 5 (51). - С. 35-37.

22. Гусева Д.Н., Курбанова Г.Я., Васильев В.В. Оценка эффективности нестационарного заводнения для послойно-неоднородных залежей при различных подвижностях нефти // Нефтепромысловое дело. - 2016. - № 4. - С. 20-23.

23. Метт Д.А., Аубакиров А.Р. Изучение движения сигнала от возмущающей скважины к наблюдательной // Экспозиция. Нефть. Газ. - 2017. - № 1 (54). - С. 26-29.

24. Владимиров И.В., Велиев Э.М., Альмухаметова Э.М. Определение оптимальных периодов работы/простоя нагнетательных скважин при нестационарном заводнении залежей высоковязкой нефти с коллектором двойной проницаемости // Проблемы сбора, подготовки и транспорта нефти и нефтепродуктов. - 2014. № 4 (98). - C. 64-74.

25. Медведев К.Ю. Перспективы применения нестационарного заводнения с целью повышения выработки запасов нефти // Наука. Инновации. Технологии. - 2017. - № 2. - С. 147-158.

26. Циклис И.М., Моисеева Е.Ф. Исследование влияния продолжительности периода простоя/работы нагнетательных скважин на эффективность применения нестационарного заводнения в нефтяных залежах в условиях частичного разгазирования // Булатовские чтения. - 2017. - Т. 2. - С. 296-301.

27. Оценка оптимальных параметров технологии нестационарного заводнения в условиях трещиноватых коллекторов месторождения Белый тигр / Т.Н. Ты, М.С. Антонов, Е.Ю. Андреев, А.Г. Гумеров // Нефтепромысловое дело. - 2013. - № 12. - С. 61-64.

28. Владимиров И.В., Альмухаметова Э.М., Варисова Р.Р. Повышение эффективности технологии нестационарного заводнения + изменения направления фильтрационного потока в результате перевода высокодебитных обводненных добывающих скважин в нестационарный режим работы // Нефтепромысловое дело. - 2016. № 5. - C. 5-9.

29. Поплыгин В.В., Уирсигроч М. Оценка эффективности применения волнового воздействия в карбонатных коллекторах с высокой вязкостью нефти // Вестник Пермского национального исследовательского политехнического университета. Геология. Нефтегазовое и горное дело. - 2018. - Т. 18. - № 2. - С. 149-156.

Поступила: 12.12 .2018 2.

\section{Информация об авторах}

Поплыгин В.В., кандидат технических наук, доцент кафедры нефтегазовых технологий Пермского национального исследовательского политехнического университета.

Уирсигроч Мариан, доктор технических наук, профессор, директор центра Прикладной динамики, Соединенное Королевство Великобритании и Северной Ирландии. 
UDC 622.276

\title{
RESEARCH OF EFFICIENCY OF COMPLEX NON-STATIONARY IMPACT ON LAYER WITH HIGH-QUALITY OIL
}

\author{
Vladimir V. Poplygin', \\ poplygin@bk.ru \\ Marian Wiercigroch², \\ m.wiercigroch@abdn.ac.uk \\ 1 Perm National Research Polytechnic University, \\ 29, Komsomolsky avenue, Perm, 614990, Russia. \\ 2 Centre for Applied Dynamics Research, \\ 69, University Rd., Aberdeen, AB24 3UE, United Kingdom of Great Britain and Northern Ireland.
}

Relevance. In case of stationary water injection to maintain reservoir pressure, a surplus irrigation of producing wells is observed. Premature watering is associated with high heterogeneity of reservoirs, high oil viscosity, bottom water and intensive water injection. The use of cyclical flooding allows increasing formation coverage of the impact and the current oil recovery rate. The increase in oil production from the use of the method reaches $4 \%$; water withdrawal from the reservoir is reduced.

The aim of the research is to investigate the efficiency of using cyclic flooding on deposits with high-viscosity oil, assess the most effective duration of the periods of operation and shutdown of injection and production wells to achieve the greatest gains in oil production.

Object: the area of carbonate reservoir with high-viscosity oil. The average water content of wells in the reservoir is $77,1 \%$, with $49 \%$ oil recovery. The site is operated by one injection well located in the center and nine producing wells along the perimeter of the oil-water contact. In the conditions under consideration, it is necessary to introduce methods to increase the coverage of the paste and reduce water content.

Methods: determination of optimal technologies for cyclic water injection and complex non-stationary effects, hydrodynamic modeling of cyclic effects on a section of an oil field.

Results. The authors have considered various options for injection well operation with implementation of cyclic flooding. It was obtained that the ratio of work duration and well shutdown, provided $100 \%$ compensation, does not practically affect the simulation result. During the cyclical operation of the injection well, the increase in oil production occurs by 3,6\% compared with the base case. Greater technological effect is possible with a complex effect - periodic shutdown of both injection and production wells. In the case of a complex effect, the cumulative oil production at the reservoir site increases by 10,6\% compared with the base case.

Key words:

Oil layer, water cut, cyclical water flooding, unsteady water flooding, oil recovery.

The research was financially supported by the of Administration of Perm region within the scientific task no. C-26/786, 21.12.2017.

\section{REFERENCES}

1. Ning J. Effects of different types of reservoir allocation and injection factors and model analysis. AIP Conference Proceedings, 9 November 2018, vol. 2036, paper 030043.

2. Liang S., Liu Y., Zhang N., Zeng B. Study on the influence of injection/production type and well pattern and spacing on producing degree of thin and poor layers. Journal of Petroleum Exploration and Production Technology, 1 September 2018, vol. 8, Iss. 3, pp. 861-870.

3. Uzoechina F., Awemo K.N., Pieve La Rosa A.E., Spyrou C., Khataniar S.K. Optimization of waterflood designs and operations using streamline simulation: application to an oil field in the North German basin. Oil Gas European Magazine, September 2018, vol. 44, Iss. 3, pp. 138-145.

4. Feng J., Luo R., Tang W., Zhang H., Liu T., Wang D. A new method for development evaluation of waterflooding reservoirs. Springer Series in Geomechanics and Geoengineering, 2019, Iss. 216039, pp. 1414-1422.

5. Wang J., Liu H., Zhang J., Meng Q., Liu H., Ge L., Zhu Z., Liu C. Experimental investigation on water flooding and continued EOR techniques in buried-hill metamorphic fractured reservoirs. Journal of Petroleum Science and Engineering, December 2018, vol. 171, pp. 529-541.

6. Bataee M., Irawan S. Porosity and permeability alteration around wellbore during injection process. International Journal of Geomechanics, 1 February 2018, vol. 18, Iss. 2, paper 04017145.

7. Lyu W., Zeng L., Chen M., Qiao D., Fan J., Xia D. An approach for determining the water injection pressure of low-permeability reservoirs. Energy Exploration and Exploitation, 1 September 2018, vol. 36, Iss. 5, pp. 1210-1228.

8. Yu K., Li K., Li Q., Li K., Yang F. A method to calculate reasonable water injection rate for $\mathrm{M}$ oilfield. Journal of Petroleum
Exploration and Production Technology, 1 December 2017, vol. 7, Iss. 4, pp. 1003-1010.

9. Ogbeiwi P., Aladeitan Y., Udebhulu D. An approach to waterflood optimization: case study of the reservoir X. Journal of Petroleum Exploration and Production Technology, 1 March 2018, vol. 8, Iss. 1, pp. 271-289.

10. Van Den Hoek P.J., Al-Masfry R., Zwarts D., Jansen J.D., Hustedt B., Van Schijndel L. Optimizing recovery for waterflooding under dynamic induced fracturing conditions. SPE Reservoir Evaluation and Engineering, October 2009, vol. 12, Iss. 5, pp. 671-682.

11. Ling Z.-F., Hu Y.-L., Li B.-Z., Wang L.-J. Optimization of horizontal injection and production pattern. Shiyou Kantan Yu Kaifa. Petroleum Exploration and Development, February 2007, vol. 34, Iss. 1, pp. 65-72.

12. Yang L. Water Injection Study in a Block Cycle. IOP Conference Series: Earth and Environmental Science, 17 July 2018, vol. 170, Iss. 2, paper 022038.

13. Savenok O.V., Povarova L.V., Gaskarov N.R. The effectiveness of chemical methods of formation stimulation and non-stationary cyclic waterflooding on the Vyngapurovskoye field. Readings name of a.i. Bulatov, 2018, vol. 2, no. 2, pp. 146-151. In Rus.

14. Tarakanova O.E., Galiullin M.M., Duboveckaya N.V. Non-stationary water injection as a way of waterflood system performing. Oil Industry, 2013, no. 11, pp. 49-53. In Rus.

15. Chikirov R.R., Mamchistova E.I., Chikirov R.R. Unsteady waterflooding as one of methods for high-viscous oil reservoirs. Advances in current natural sciences, 2018, no. 8, pp. 191-196. In Rus.

16. Gulyaev V.N., Kiprin I.I., Zakharova N.P. Application of nonstationary technology to increase oil recovery factor of fields' areas 
with hard-to-recover oil reserves. Geology, geophysics and development of oil and gas fields, 2015, no. 10, pp. 39-44. In Rus.

17. Yartiev A.F., Khabibrakhmanov A.G., Podavalov V.B., Bakirov A.I Cyclic water flooding of Bobric formation at Sabanchinskoye field. Oil Industry, 2017, no. 3, pp. 85-87. In Rus.

18. Vladimirov I.V., Veliev E.M., Almukhametova E.M., Abilkhairov D.T. Application of non-stationary flooding for extra-viscous oil from the collector with dual permeability. Theory.Problems of gathering, treatment and transportation of oil and oil products, 2014, no. 4 (98), pp. 16-25. In Rus.

19. Veliev E.M. Efficiency of non-stationary flooding as a function of injection wells idle time for the highly productive light oil reservoirs Problems of gathering, treatment and transportation of oil and oil products, 2015, no. 2 (100), pp. 46-56. In Rus.

20. Chumakov G.N. Probabilistic estimate of effectiveness of the method of cyclic bed fluid injection. Bulletin of PNRPU. Geology. Oil \& Gas Engineering \& Mining, 2014, vol. 13, no. 13, pp. 49-58. In Rus.

21. Pyatibratov P.V., Aubakirov A.R. Assessing the impact of reservoir permeability anisotropy on the cyclic waterflooding effectiveness. Exposition Oil Gas, 2016, no. 5 (51), pp. 35-37. In Rus.

22. Guseva D.N., Kurbanova G.Ya., Vasilev V.V. Evaluation of nonstationary water-flooding efficiency for layer-by-layer heterogeneous deposits in conditions of different oil mobility. Neftepromyslovoe delo, 2016, no. 4, pp. 20-23. In Rus.

23. Mett D.A., Aubakirov A.R. The study of signal changes dynamics from disturbing well to observation well. Exposition Oil Gas, 2017, no. 1 (54), pp. 26-29. In Rus.
24. Vladimirov I.V., Veliev E.M., Almukhametova E.M. Determination of the optimal operation/shutdown periods of injection wells under nonstationary flooding of highly viscous oil deposits with reservoir dual permeability. Problems of gathering, treatment and transportation of oil and oil products, 2014, no. 4 (98), pp. 64-74. In Rus.

25. Medvedev K.Yu. Prospects for the application of non-stationary waterflooding to increase the production of oil reserves. Nauka. Innovatsii. Tekhnologii, 2017, no. 2, pp. 147-158. In Rus.

26. Tsiklis I.M., Moiseeva E.F. The study of the effect of time of stopping/operating period of injection wells on the effectiveness of non-stationary flooding in partially degassed deposits. Readings name of a.i. Bulatov, 2017, vol. 2, pp. 296-301. In Rus.

27. Ty T.N., Antonov M.S., Andreev E.Yu., Gumerov A.G. Assessment of optimal parameters of non-stationary water-flooding technology in conditions of collectors' fracturing of White Tiger field. Neftepromyslovoe delo, 2013, no. 12, pp. 61-64. In Rus.

28. Vladimirov I.V., Almukhametova E.M., Varisova R.R. Efficiency enhancement of non-stationary water-flooding technology with the change of filtration flow direction due to conversion of high-flow-rate water-flooded wells into a non-stationary operational. Neftepromyslovoe delo, 2016, no. 5, pp. 5-9. In Rus.

29. Poplygin V.V., Wiercigroch M. Evaluation of the wave effect in carbonate reservoirs with high viscosity oil. Perm Journal of Petroleum and Mining Engineering, 2018, vol. 18, no. 2, pp. 149-156. In Rus.

Received: 12 December 2018.

\section{Information about the authors}

Vladimir V. Poplygin, Cand. Sc., associate professor, Perm national Research Polytechnic University.

Marian Wiercigroch, Dr. Sc., director of the Centre for Applied Dynamics Research, University of Aberdeen. 